\title{
Evolution and taxonomy of native mesorhizobia nodulating medicinal Glycyrrhiza species in
} China

$\underline{\text { Seyed Abdollah Mousavi }}{ }^{1 * \dagger}, \mathrm{Li} \mathrm{Li}^{2,3 \dagger}$, Gehong $\mathrm{Wei}^{4}$, Leena Räsänen ${ }^{2}$, Kristina Lindström ${ }^{1}$

${ }^{1}$ University of Helsinki, Department of Environmental Sciences, Helsinki, Finland.

${ }^{2}$ University of Helsinki, Department of Food and Environmental Sciences, Helsinki, Finland.

${ }^{3}$ Key Laboratory of Biogeography and Bioresource in Arid Land, Xinjiang Institute of Ecology and Geography, Chinese Academy of Sciences, Urumqi, 830011, China.

${ }^{4}$ Northwest A\&F University, College of Life Sciences, Yangling, Shaanxi, China.

*Corresponding author. email address: $\quad$ s.abdollah.mousavi@gmail.com or seyed.mousavi@helsinki.fi (Seyed Abdollah MOUSAVI).

†The authors wish it to be known that, in their opinion, the first two authors should be regarded as Joint First Authors.

Department of Environmental Sciences, Viikinkaari 2a, P.O. Box 65, University of Helsinki, Helsinki, Finland, FIN-00014.

Running title: Phylogeny of mesorhizobia nodulating Glycyrrhiza

\section{Abstract}

Previously, 159 bacterial strains were isolated from the root nodules of wild perennial Glycyrrhiza legume species grown on 40 sites in central and north-western China, in which 57 strains were classified as "true symbionts" belonging to the genus Mesorhizobium based on amplified fragment length polymorphism (AFLP) genomic fingerprinting and partial sequences of the 16S rRNA gene [20]. In the present work, the phylogeny of Glycyrrhiza nodulating mesorhizobia was further examined by multilocus sequence analysis (MLSA). The concatenated gene tree of three housekeeping genes (16S rRNA, recA, and rpoB) of 59 strains including the 29 mesorhizobial test strains and 30 type mesorhizobial species, was constructed applying the maximum likelihood method and Bayesian inference. In the concatenated gene tree, the 29 test strains were distributed in seven separate clades. Seventeen test strains clustered with $M$. tianshanense, M. temperatum, M. muleiense, and $M$. alhagi with high bootstrap support $(\mathrm{BS}>85 \%)$. Eight test strains did not cluster with any of the described Mesorhizobium species. Based on the results, we proposed these eight test strains might 
belong to a putative new species of the genus Mesorhizobium. The sequences of three accessory genes (nodA, nodC, and nifH) of the test strains were also analysed and were compared with those of representatives of the 30 described mesorhizobial species. The results showed that mesorhizobia involved in symbiosis with Glycyrrhiza plants probably have acquired some genetic material from other rhizobia in co-evolution with Glycyrrhiza and other legume species.

Key words: MLSA, Housekeeping genes, Accessory genes, Mesorhizobium, Glycyrrhiza.

\section{Introduction}

The plant genus Glycyrrhiza belongs to the family Fabaceae/Leguminosae and consists of approximately 20 species, among which six species produce a sweet saponin glycyrrhizic acid (glycyrrhizin). Roots and rhizomes of Glycyrrhiza glabra, G. uralensis and G. inflata are considered as licorice, and are used extensively in food, tobacco, cosmetics, and medicine industries [17, 20]. Glycyrrhiza species can be found on all continents but Antarctica; and are mostly endemic to Eurasia. G. glabra is a Mediterranean species which grows also in Iran, Iraq, Central Asia and the northwestern part of China, whereas the species G. uralensis is found in China, Central Asia, and Mongolia. Glycyrrhiza spp. grow on dry grassy plains and mountainsides in the north-western provinces of China. Even though medicinal properties of Glycyrrhiza spp. were studied extensively, over 1500 reports exist on the compound glycyrrhizin, the biology and ecology of the plant species are not well known [15, 14, 20, 33].

Glycyrrhiza spp. can establish symbiotic associations with a wide range of root-nodule bacteria, which in symbiosis reduce atmospheric dinitrogen gas $\left(\mathrm{N}_{2}\right)$ to ammonia. At least three symbiotic rhizobial genera, Mesorhizobium, Ensifer (syn. Sinorhizobium) and Rhizobium were found to be associated with Glycyrrhiza spp. [20]. The genera Rhizobium and Ensifer are classified in the family Rhizobiaceae whereas the genus Mesorhizobium consisting of 30 species is the member of the family Phyllobacteriaceae (http://www.bacterio.net).

Li et al. [20] studied 159 endophytic bacterial isolates form nodules of wild Glycyrrhiza growing on 40 sites in central and north-western China. The results of amplified fragment length polymorphism (AFLP) genomic fingerprinting, and partial sequences of the 16S rRNA gene as well as nodulation tests indicated that out of 159 endophytic strains 115 represented Alphaproteobacteria, involving the genera Mesorhizobium (57 strains), Rhizobium (25), Ensifer (Sinorhizobium) (11), Phyllobacterium (6) and Agrobacterium (16). Thus, at least, representatives of all these genera can colonize 
Glycyrrhiza nodules in China. Based on symbiotic properties, five Mesorhizobium groups (with 57 strains) represented the true symbionts of G. glabra and G. uralensis. Since the 16S rRNA gene is too conserved to allow close species separation $[22,23,24]$, and only partial sequences of $16 \mathrm{~S}$ rRNA gene ( $r r s)$ of representative strains of each AFLP subgroups were studied by Li et al. [20], further phylogenetic studies were required to resolve the taxonomy of these isolates. Multilocus sequence analysis (MLSA) method is considered as a straightforward method to study the phylogeny and taxonomy of rhizobia [22, 23, 24, 25].

Aiming at determining the accurate taxonomic position of 29 mesorhizobial strains collected from nodules of Glycyrrhiza spp. growing in central and north-western China, we performed multilocus sequence analysis (MLSA) for 59 mesorhizobial strains, including 29 Glycyrrhiza test strains and 30 type strains of the Mesorhizobium species, since multilocus sequence analysis (MLSA) method is considered as a straightforward method to study the phylogeny and taxonomy of rhizobia [22, 23, 24, 25]. Three housekeeping genes, namely $16 \mathrm{~S}$ rRNA ( $r r s$ ), recA and $r p o B$, coding for $16 \mathrm{~S}$ ribosomal RNA, recombinase A, and RNA polymerase beta subunit, respectively were used in this study. Furthermore, we also investigated the phylogeny of three symbiotic genes of all the 59 mesorhizobial strains, involving nodulation genes nodA and nodC and the nitrogenase encoding gene nifH.

\section{Material and Methods}

\section{Bacterial strains and DNA isolations}

59 mesorhizobial strains studied here (Table S1) included 29 mesorhizobial strains isolated from Glycyrrhiza glabra (10 strains), G. uralensis (14) and unidentified Glycyrrhiza species (5), and 30 type strains of the Mesorhizobium species, in which Mesorhizobium camelthorni HAMBI $3020^{\mathrm{T}}, M$. muleiense HAMBI $3264^{\mathrm{T}}$, M. qingshengii HAMBI $3277^{\mathrm{T}}$, M. robiniae HAMBI $3082^{\mathrm{T}}$, M. sangaii HAMBI $3318^{\mathrm{T}}$ and $M$. shangrilense HAMBI $3050^{\mathrm{T}}$ were obtained from the HAMBI Culture Collection, University of Helsinki. The bacterial samples were grown on yeast mannitol agar medium at $28^{\circ} \mathrm{C}$ for two-three days [27]. For DNA isolation, single colonies of the bacteria were cultured in 5 $\mathrm{ml}$ of tryptone-yeast extract broth. Genomic DNA was isolated from each strain using the UltraClean Microbial DNA Isolation Kit (MO BIO Laboratories, Inc.) and was preserved at $-20^{\circ} \mathrm{C}$.

\section{PCR amplification and gene sequencing}

Three housekeeping genes (rrs-recA-rpoB) and three symbiotic genes (nodA, nodC and nifH) of the Glycyrrhiza test strains and the $r р о B$ gene of six reference strains (not available in database) were sequenced in the current work. PCR amplification and sequencing were performed by using the 
primers listed in Table S2. The sequences of the mentioned genes for most of the type strains of Mesorhizobium species were obtained from GenBank (http://www.ncbi.nlm.nih.gov/genbank). The accession numbers of the studied housekeeping and symbiotic gene sequences are listed in Table S1.

\section{Phylogenetic analyses of the sequences}

The sequences were aligned by MUSCLE software at EML-EBI [5] and were edited with program BioEdit version 7.0.5.3 [10]. The best-fit nucleotide substitution model of each gene was selected applying MEGA6 [29]. The concatenated gene tree ( $r r s-a t p D-r p o B)$ of altogether 59 mesorhizobial strains was constructed using maximum likelihood (ML) and Bayesian interference. The ML tree of the combined genes (rrs-atpD-rpoB) was constructed with 1,000 bootstrap replicates in MEGA6. The mean distance between the groups of the test and reference strains and the distances between the species were computed by MEGA6. The algorithm Metropolis-coupled Markov chain Monte Carlo (MCMCMC) for $2 \times 10^{6}$ generations was run twice with MrBayes 3.2 for the combined sequences dataset [26]. The generated Bayesian traces and trees were visualized by Tracer v1.5 (http://tree.bio.ed.ac.uk/software/tracer/) $\quad$ and $\quad$ FigTree v1.4.0 (http://tree.bio.ed.ac.uk/software/figtree/). The ML individual gene trees of the 16S rRNA, nodA, nodC, and nifH loci were constructed with 1,000 bootstrap replicates in MEGA6.

\section{Results}

\section{Analyses of the housekeeping genes}

The phylogeny of the $r r s$ gene sequence (1247 bp) of 59 mesorhizobial strainsdemonstrated that the 29 mesorhizobial test strains were placed in five different clusters (Fig. S1). The ML tree of the combined three housekeeping genes (rrs-recA-rpoB) (Fig. 1) positioned the 29 test strains in seven separate clades. In addition, the Bayesian analysis returned similar topologies (not shown).

Eight strains isolated from Xinjiang region (Chahan, Hejing, and Ziniquan towns) formed a distinct clade (clade $\mathrm{C}$ ) with a high bootstrap support $(\mathrm{BS}=100 \%)$ that accommodates none of the reference strains. Seventeen Chinese test strains clustered with M. tianshanense, M. temperatum, M. muleiense, and $M$. alhagi with high confidence (BS>85\%), representing the groups A, D, E, and G respectively. The strain NWGS09 was placed close to $M$. tianshanense and $M$. gobiense. Based on the sequences of two protein-coding housekeeping genes (recA-rрoB), the pairwise ANI between eight Mesorhizobium species were over 96\%. The strain NWSX24 was placed close to the clade that accommodates $M$. temperatum and three test strains (clade D). The strain NWNX05, isolated from Glycyrrhiza sp. in Ningxia, was grouped with M. gobiense supported by $100 \%$ of bootstrap value. Strain NWGS05, isolated from Glycyrrhiza sp. in Lingtai, Gansu, was clustered with M. amorphae ( $\mathrm{BS}=100 \%$ ). Based on the sequences of two protein-coding housekeeping genes (recA-rpoB), the pairwise ANI between the following four pairs of species, M. qingshengii and M. huakuii, M. 
silamurunense and $M$. shonense, M. tianshanense and M. gobiense, and M. alhagi and M. camelthorni were over $96 \%$.

\section{Analyses of the individual accessory genes}

Based on the analyses of the nodA gene sequences (Fig. S2 and Table 1), 28 test strains were placed in four main groups (A, B, C and E). Interestingly, the strains of clade B were placed separately from the reference strains. The nodA sequences of the species $M$. tianshanense and M. temperatum were grouped with 15 test strains, though neither of the clusters was strongly bootstrapping supported (BS<75\%). In the nodA gene tree, the species $M$. alhagi formed a well-supported clade (E) with five Chinese test strains alongside the species M. camelthorni.

In the nodC gene tree, 28 Chinese test strains were positioned in seven clusters (A-G). The clades A, B, E, and $\mathrm{G}$ accommodated the type strains of the species M. tianshanense, M. gobiense, M. temperatum, M. camelthorni and M. alhagi. In the nifH gene tree, the Chinese test strains were placed in nine lineages. Clade A was positioned close to M. tianshanense and strain NWGS09.In the three accessory gene trees, the Chinese test strains did not cluster with the same reference species, with which they were grouped in the concatenated housekeeping gene tree. Among the type strains, the species $M$. alhagi was positioned close to M. camelthorni in the three studied accessory gene trees.

\section{Discussion}

The results of the present MLSA of three housekeeping genes (rrs-recA-rpoB) demonstrated that 20 test strains isolated mostly from the plant species G. glabra and G. uralensis were placed in the clades A, B, D, E, F, and G, which represent the species $M$. tianshanense, $M$. gobiense, $M$. temperatum, $M$. muleiense, M. amorphae, M. alhagi, and M. camelthorni, respectively. All above mentioned seven reference species were originally isolated in China, from a wide range of legumes. Eight test strains representing clade C, namely NWXJ21, NWXJ27, NWXJ31, NWXJ 33, NWXJ36, NWJ39, NWXJ40, and NWXJ43, did not cluster with any of the 30 described Mesorhizobium species, implying that they could belong to a putative novel species of the genus Mesorhizobium.

Konstantinidis et al. [16] proposed an ANI of 96\% corresponds to a cut-off of $70 \%$ DNA-DNA hybridization for species delineation, whilst Martens et al. [22] proposed an ANI of $97.3 \%$ for species delineation within the genus Ensifer. Moreover, the study by Figueras et al. [6] recommended a cut- 
off score of $95 \%$ for species delineation. These variations demonstrated that the cut-off ANI values may change when different genes were used. The concatenated gene tree and the ANI analyses in this study showed that the following four pairs of species, M. qingshengii-M. huakuii, M. silamurunenseM. shonense, M. tianshanense-M. gobiense, and M. alhagi-M. camelthorni might need to be merged into four species. However, it is worth noting that in this study, we only analysed three housekeeping genes, which might not be a sufficient number of genes to delineating new species and species combinations. Merging these eight species requires a more comprehensive phylogenetic study of mesorhizobia preferably by comparing whole-genome sequences. In the concatenated housekeeping gene tree, strain NWGS09, which was isolated from Glycyrrhiza sp. in Gansu, was positioned between the two species $M$. tianshanense and $M$. gobiense. However, since the two species $M$. tianshanense and M. gobiense are very similar to each other, strain NWGS09 cannot represent a new species. The results of MLSA confirmed the groups formed based on AFLP, a whole-genome fingerprinting method, by $\mathrm{Li}$ et al. [20], with the exception of the grouping of strains NWSX22, NWGS09, and NWNX05.

In the nodA gene tree, 15 test strains (clades $\mathrm{A}$ and $\mathrm{C}$ ) were clustered with two species, $M$. tianshanense and M.temperatum. The former species was isolated from G. uralensis in the Xinjiang of China and the latter one was isolated from Astragalus adsurgens in northern regions of China [3, 7]. However, Based on the nodC sequences, the 15 test strains (clades A, B, E, and G) clustered with the species $M$. tianshanense, $M$. gobiense, M. temperatum, M. camelthorni, and M. alhagi. The species $M$. tianshanense, $M$. gobiense, $M$. temperatum, $M$. alhagi, and $M$. camelthorni are native species of China. The species $M$. alhagi, and M. camelthorni were isolated from the plant genus Alhagi in Xinjiang, and M. gobiense was isolated form the genus Oxytropus. However, the two species M. alhagi, and M. camelthorni were also able to nodulate Glycyrrhiza inflata and M. gobiense could form nodules on $G$. uralensis respectively $[1,2,11]$. In the nodA and nodC gene trees, several test strains were grouped close to the species $M$. tianshanense, which was also isolated from the plant genus Glycyrrhiza in China [3].

The phylogeny of symbiotic genes is often incongruent with the housekeeping genes $[28,31]$. This was also observed in our study for most of the strains isolated from Glycyrrhiza species. Albeit the representatives of clades $\mathrm{A}, \mathrm{C}$ and $\mathrm{G}$ of the combined housekeeping gene tree grouped together also in the nodA, nodC and nifH gene trees, the positions of the clades in the three symbiotic gene trees and the concatenated housekeeping gene tree were not similar, indicating that in the case of mesorhizobia, the phylogeny of the tested accessory genes were not congruent with housekeeping genes in our study. 
Three strains of clade E (NWGS01, NWGS03, NWSX06) of the MLSA tree were grouped together in the nodA, nodC and nifH gene trees. In the concatenated housekeeping gene tree, these three strains formed a monophyletic group (clade E) with two other test strains (NWSX22 and NWSX23) and $M$. muleiense. However, these five test strains were not clustered together in the accessory gene trees. The species M. muleiense was placed within the clade E (MLSA), and close to the species $M$. temperatum in the concatenated gene tree, whereas, in the nodC and nifH gene trees, M. muleiense was placed distinctly from the other strains of clade E (MLSA) and M. temperatum (the sequence of nodA gene is missing for M. muleiense). Strain NWSX19 (representing MLSA clade D) appeared more similar to NWSX22 (representing MLSA clade E) in the nodA and nodC gene trees, but not in the nifH gene tree.

To find out whether the variation in genomic fingerprint AFLP patterns exhibits specific biogeographical patterns, Li et al. [20] performed multivariate analyses of AFLP patterns of 159 bacterial strains isolated from nodules of Glycyrrhiza spp. in China. It showed that plant species and geographic location explained only a small part of the total variation in AFLP patterns (14.4\%). However, strains isolated from G. glabra were clearly separated from those obtained from $G$. uralensis, and the strains obtained from Central China were well separated from those originating Xinjiang, indicating host preference and regional endemism [20].

In the current work, the clades of the combined housekeeping gene tree accommodated strains isolated from the same regions, with the exception of clade E. The clade E contains strains from two different regions, Gansu and Shaanxi, which are neighbor provinces. In the case of the strains of clade E which were isolated from the plant G. uralensis in two neighbor provinces (Shaanxi and Gansu), three strains (NWGS01, NWGS03, and NWSX06) were placed distinctly from (NWSX22 and NWSX23) in the accessory gene trees. All strains isolated from the same provinces did not form a monophyletic lineage either in the combined housekeeping gene tree or in the symbiotic gene trees. Wernegreen and Riley [31] also reported that geographical positions of rhizobial strains have a relatively poor fit across their phylogenies at the species level in comparison with host plant genus. Glycyrrhiza glabra grows mainly in Xinjiang (the northwest), whereas G. uralensis grows in all parts of northern China [15]. In the study of $\mathrm{Li}$ et al. [20], the geographic distribution of these two Glycyrrhiza species sampled was similar. Based on genetic markers, Kondo et al. [15] concluded that G. glabra is more closely related to G. inflata than to G. uralensis, G. glabra and G. inflata being equidistant to G. uralensis. However, the genetic difference between them is not large because $G$. uralensis can hybridize with $G$. glabra and $G$. inflata $[13,15]$. This close genetic relationship is supposed to explain why most nodulating mesorhizobial strains were capable of forming nodules on both G. uralensis and G. glabra [20]. Unlike the report on mesorhizobia isolated from Acacia and 
Sesbania sesban tree species [4], where mesorhizobia isolated from the same plant were grouped together in MLSA based on housekeeping genes ( $g l t A, g y r B$, rec $A$, and $r p o B$ ), clustering of the strains isolated from Glycyrrhiza was not observed in this work. The differences between these two results might be due to the plant genera Acacia (Mimosoideae) and Sesbania (Papilioinoideae) being distantly related, whilst G. glabra and G. uralensis have a very close relationship and share similar growth conditions.

At a higher phylogenetic level, in the symbiotic gene trees, most of the mesorhizobia isolated from China were placed separately from the Mesorhizobium species isolated from other countries than China. The plant species G. glabra is a Mediterranean species, and the species G. uralensis is probably originated from the northern or north-western neighbours of China. Therefore, the Chinese mesorhizobia involved in symbiosis with the plant of $G$. uralensis and G. uralensis might have acquired some genetic material from other rhizobia in co-evolution with Glycyrrhiza and other legume species. Probably, the same Mesorhizobium species can induce nitrogen-fixing nodules on many host plants. A similar hypothesis was proposed by Tan et al. [30] about the co-evolution of some rhizobia and Carmichaelinae plants in New Zealand. Likewise, Wernegreen and Riley [31] pointed out that mapping of host plant genus across the Mesorhizobium nod phylogeny is less clear in comparison with the genera Rhizobium and Ensifer. Based on the phylogenetic analyses of the three symbiotic genes, the Chinese test strains were grouped with bacteria that were isolated from the plant genera Alhagi, Astragalus, Amorpha, Oxytropis, Glycyrrhiza and Caragana in China, and from the genus Anthyllis in France. These genera belong to the inverted repeat-lacking clade (IRLC) of legumes; with the exception of the genera Amorpha and Anthyllis, which are positioned in the plant clades Dalbergioids s.l. and Robinioids [21, 32]. However, the close relationships of the plants could affect that several Mesorhizobium species can form at least non-fixing nodules on these plants.

To conclude, the current work showed that eight Mesorhizobium strains isolated from G. uralensis and G. glabra in central and north-western China belong to a putative new species. Whole-genome sequencing of more mesorhizobial species will contribute to ascertaining the taxonomic positions of the members of the genus Mesorhizobium. A genealogy of rhizobia from the gene center of Glycyrrhiza could reveal more about the co-evolution of mesorhizobia and Glycyrrhiza.

\section{Acknowledgments}

We would like to thank Riitta Saastamoinen for providing us the bacterial strains used in this study. This research was funded by the Academy of Finland, Societas Scientiarum Fennica, the Department of Environmental Sciences of the University of Helsinki, and the National Science Foundation of China, and the 973 project of China (2010CB126502). 


\section{References}

[1] Chen, W., Zhu, W., Bontemps, C., Young, J.P.W., Wei, G.H. (2010). Mesorhizobium alhagi sp. nov., isolated from wild Alhagi sparsifolia in north-western China. Int. J. Syst. Evol. Microbiol. 60,958-962 .

[2] Chen, W., Zhu, W., Bontemps, C., Young, J.P.W., Wei, G. (2011). Mesorhizobium camelthorni sp. nov., isolated from Alhagi sparsifolia. Int. J. Syst. Evol. Microbiol. 61,574-579

[3] Chen, W., Wang, E., Wang, S., Li, Y., Chen, X., Li, Y. (1995). Characteristics of Rhizobium tianshanense sp. nov., a Moderately and Slowly Growing Root Nodule Bacterium Isolated from an Arid Saline Environment in Xinjiang, People's Republic of China. Int. J. Syst. Evol. Microbiol. 45,153-159.

[4] Degefu, T., Wolde-meskel, E., Frostegård, Å. (2011). Multilocus sequence analyses reveal several unnamed Mesorhizobium genospecies nodulating Acacia species and Sesbania sesban trees in Southern regions of Ethiopia. Syst. Appl. Microbiol. 34,216-226

[5] Edgar, R.C. (2004) MUSCLE: multiple sequence alignment with high accuracy and high throughput. Nucleic Acids Res. 32, 1792-1797.

[6] Figueras, M.J., Beaz-Hidalgo, R., Hossain, M.J., Liles, M.R. (2014). Taxonomic Affiliation of New Genomes Should Be Verified Using Average Nucleotide Identity and Multilocus Phylogenetic Analysis. Genome Announc. 2,e00927-14.

[7] Gao, J., Turner, S.L., Kan, F.L., Wang, E.T., Tan, Z.Y., Qiu, Y.H., Gu, J., Terefework, Z., Young, J.P.W., Lindström, K., Chen, W.X. (2004). Mesorhizobium septentrionale sp. nov. and Mesorhizobium temperatum sp. nov., isolated from Astragalus adsurgens growing in the northern regions of China. Int. J. Syst. Evol. Microbiol. 54,2003-2012.

[8] Gaunt, M.W., Turner, S.L., Rigottier-Gois, L., Lloyd-Macgilp, S.A., Young, J.P. (2001) Phylogenies of atpD and recA support the small subunit rRNA-based classification of rhizobia. Int. J. Syst. Evol. Microbiol. 51, 2037-2048.

[9] Goujon, M., McWilliam, H., Li, W., Valentin, F., Squizzato, S., Paern, J., Lopez, R. (2010) A new bioinformatics analysis tools framework at EMBL-EBI. Nucleic Acids Res. 38, W695-W699. [10] Hall T.A., (1999) BioEdit: a user-friendly biological sequence alignment editor and analysis program for Windows 95/98/NT. Nucl. Acid. Symp. Ser. 41, 95-98.

[11] Han, T.X., Han, L.L., Wu, L.J., Chen, W.F., Sui, X.H., Gu, J.G., Wang, E.T., Chen, W.X. (2008). Mesorhizobium gobiense sp. nov. and Mesorhizobium tarimense sp. nov., isolated from wild legumes growing in desert soils of Xinjiang, China. Int. J. Syst. Evol. Microbiol. 58,2610-2618.

[12] Haukka, K., Lindström, K., Young, J.P. (1997). Three Phylogenetic Groups of nodA and nifH Genes in Sinorhizobium and Mesorhizobium Isolates from Leguminous Trees Growing in Africa and Latin America. Appl. Environ. Microbiol. 64,419-426.

[13] Hayashi, H., Hattori, S., Inoue, K., Sarsenbaev, K., Ito, M., Honda, G. (2003). Field survey of Glycyrrhiza plants in central Asia (1). Characterization of G. uralensis, G. glabra and the putative intermediate collected in Kazakhstan. Biol. Pharm. Bull. 26,867-871.

[14] Hayashi, H. Sudo, H. (2009). Economic importance of licorice. Plant. Biotechnol. 26,101-104

[15] Kondo, K., Shiba, M., Yamaji, H., Morota, T., Zhengmin, C., Huixia, P., Shoyama, Y. (2007). Species identification of licorice using nrDNA and cpDNA genetic markers. Biol. Pharm. Bull. 30,1497-1502

[16] Konstantinidis, K.T., Ramette, A., Tiedje, J.M. (2006). Toward a More Robust Assessment of Intraspecies Diversity, Using Fewer Genetic Markers. Appl. Environ. Microbiol. 72,7286-7293.

[17] Kushiev, H., Noble, A.D., Abdullaev, I., Toshbekov, U. (2005). Remediation of abandoned saline soils using Glycyrrhiza glabra: a study from the Hungry Steppes of Central Asia. Iint. J. Agr. Sustain. 3,102-113. 
[18] Laguerre, G., Nour, S.M., Macheret, V., Sanjuan, J., Drouin, P., Amarger, N. (2001). Classification of rhizobia based on nodC and nifH gene analysis reveals a close phylogenetic relationship among Phaseolus vulgaris symbionts. Microbiol. 147,981-993.

[19] Laranjo, M., Alexandre, A., Oliveira, S. (2014). Legume growth-promoting rhizobia: an overview on the Mesorhizobium genus. Microbiol. Res. 169,2-17.

[20] Li, L., Sinkko, H., Montonen, L., Wei, G., Lindström, K., Räsänen, L.A. (2012). Biogeography of symbiotic and other endophytic bacteria isolated from medicinal Glycyrrhiza species in China. FEMS Microbiol. Ecol. 79,46-68.

[21] Lock M. \& Schrire, B.D. 2005. Tribe Galegeae. In: Lewis, G.P. et al. (Eds.), Legumes of the World, Royal Botanic Gardens, Kew, pp. 475-487.

[22] Martens, M., Dawyndt, P., Coopman, R., Gillis, M., De Vos, P., Willems, A. (2008). Advantages of multilocus sequence analysis for taxonomic studies: a case study using 10 housekeeping genes in the genus Ensifer (including former Sinorhizobium). Int. J. Syst. Evol. Microbiol. 58,200-214 .

[23] Martens, M., Delaere, M., Coopman, R., De Vos, P., Gillis, M., Willems, A. (2007). Multilocus sequence analysis of Ensifer and related taxa. Int. J. Syst. Evol. Microbiol. 57,489-503. [24] Mousavi, S.A., Österman, J., Wahlberg, N., Nesme, X., Lavire, C., Vial, L., Paulin, L., De Lajudie, P., Lindström, K. (2014). Phylogeny of the Rhizobium-Allorhizobium-Agrobacterium clade supports the delineation of Neorhizobium gen. nov. Syst. Appl. Microbiol. 37,208-215.

[25] Mousavi, S.A., Willems, A., Nesme, X., de Lajudie, P., Lindström, K. (2015). Revised phylogeny of Rhizobiaceae: Proposal of the delineation of Pararhizobium gen. nov., and 13 new species combinations. Syst. Appl. Microbiol. 38,84-90.

[26] Ronquist, F., Teslenko, M., van der Mark, P., Ayres, D.L., Darling, A., Höhna, S., Larget, B., Liu, L., Suchard, M.A., Huelsenbeck, J.P. (2012) MrBayes 3.2: Efficient Bayesian Phylogenetic Inference and Model Choice across a Large Model Space. Syst. Biol. 61, 539-42.

[27] Somasegaran, P., and Hoben, H.J. (2012). Handbook for rhizobia: methods in legumeRhizobium technology Springer Science \& Business Media).

[28] Suominen, L., Roos, C., Lortet, G., Paulin, L., Lindström, K. (2001). Identification and structure of the Rhizobium galegae common nodulation genes: evidence for horizontal gene transfer. Mol. Biol. Evol. 18,907-916.

[29] Tamura, K., Stecher, G., Peterson, D., Filipski, A., Kumar, S. (2013). MEGA6: Molecular Evolutionary Genetics Analysis version 6.0. Mol. Biol. Evol. 30,2725-2729.

[30] Tan, H.W., Weir, B.S., Carter, N., Heenan, P.B., Ridgway, H.J., James, E.K., Sprent, J.I., Young, J.P.W., Andrews, M. (2012). Rhizobia with 16S rRNA and nifH similar to Mesorhizobium huakuii but Novel recA, glnII, nodA and nodC genes are symbionts of New Zealand Carmichaelinae. [31] Wernegreen, J.J. Riley, M.A. (1999). Comparison of the evolutionary dynamics of symbiotic and housekeeping loci: a case for the genetic coherence of rhizobial lineages. Mol. Biol. Evol. 16,98113.

[32] Wojciechowski, M.F., Lavin, M., Sanderson, M.J. (2004). A phylogeny of legumes (Leguminosae) based on analysis of the plastid matK gene resolves many well-supported subclades within the family. Am. J. Bot. 91,1846-1862.

[33] Zimnitskaya, S. (2009). State of the reproductive system of populations of species of the genus Glycyrrhiza L.(Fabaceae). Contemp. Probl. Ecol. 2,392-395. 
Table 1. List of the mesorhizobial test strains studied, plant and place of isolation, and the position of the test strains in the combined gene tree, AFLP, and the individual accessory gene trees.

\begin{tabular}{|c|c|c|c|c|c|c|c|}
\hline Strain & Host & Isolation site* & MLSA & AFLP*** & nodA & nodC & nifH \\
\hline NWXJ16 & G. uralensis & Xinjiang, Bachu & $\mathrm{G}$ & $4 a$ & $\mathrm{E}$ & $\bar{G}$ & $-* * * *$ \\
\hline NWXJ42 & G. uralensis & Xinjiang, Hejing & G & $4 a$ & E & G & I \\
\hline NWXJ02 & G. uralensis & Xinjiang, Bachu & G & $4 a$ & $\mathrm{E}$ & G & I \\
\hline NWXJ08 & G. uralensis & Xinjiang, Bachu & G & $4 a$ & $\mathrm{E}$ & G & I \\
\hline NWXJ18 & G. uralensis & Xinjiang, Bachu & G & $4 a$ & $\mathrm{E}$ & G & I \\
\hline NWXJ33 & G. glabra & Xinjiang, Chahan & $\mathrm{C}$ & $5 e$ & B & $\mathrm{C}$ & A \\
\hline NWXJ39 & G. glabra & Xinjiang, Chahan & $\mathrm{C}$ & $5 e$ & B & $\mathrm{C}$ & A \\
\hline NWXJ43 & G. uralensis & Xinjiang, Hejing & $\mathrm{C}$ & $5 e$ & B & $\mathrm{C}$ & A \\
\hline NWXJ36 & G. glabra & Xinjiang, Chahan & $\mathrm{C}$ & $5 e$ & B & $\mathrm{C}$ & A \\
\hline NWXJ27 & G. glabra & Xinjiang, Ziniquan & $\mathrm{C}$ & $5 \mathrm{e}$ & B & $\mathrm{C}$ & A \\
\hline NWXJ31 & G. glabra & Xinjiang, Ziniquan & $\mathrm{C}$ & $5 \mathrm{e}$ & B & $\mathrm{C}$ & A \\
\hline NWXJ21 & G. glabra & Xinjiang, Ziniquan & $\mathrm{C}$ & $5 e$ & B & $\mathrm{C}$ & A \\
\hline NWXJ40 & G. glabra & Xinjiang, Chahan & $\mathrm{C}$ & $5 e$ & B & $\mathrm{C}$ & A \\
\hline NWGS01 & G. uralensis & Gansu, Huanxian & $\mathrm{E}$ & $3 c$ & A & $\mathrm{F}$ & B \\
\hline NWGS03 & G. uralensis & Gansu, Huanxian & E & $3 c$ & A & $\mathrm{F}$ & B \\
\hline NWSX06 & G. uralensis & Shaanxi, Ziwuling & $\mathrm{E}$ & $3 \mathrm{c}$ & A & $\mathrm{F}$ & B \\
\hline NWSX22 & G. uralensis & Shaanxi, Yangling & $\mathrm{E}$ & $5 b$ & $\mathrm{C}$ & $\mathrm{D}$ & $\mathrm{D}$ \\
\hline NWSX23 & G. uralensis & Shaanxi, Yangling & E & $3 c$ & $\mathrm{C}$ & B & $\mathrm{D}$ \\
\hline NWSX19 & Glycyrrhiza sp. & Shaanxi, Yanan & $\mathrm{D}$ & $3 a$ & $\mathrm{C}$ & $\mathrm{D}$ & $\mathrm{F}$ \\
\hline NWSX20 & Glycyrrhiza sp. & Shaanxi, Yanan & $\mathrm{D}$ & $3 a$ & $\mathrm{C}$ & $\mathrm{E}$ & G \\
\hline NWSX24 & G. uralensis & Shaanxi, Yangling & $\mathrm{D}$ & $3 a$ & $\mathrm{C}$ & A & E \\
\hline NWSX25 & G. uralensis & Shaanxi, Yangling & $\mathrm{D}$ & $3 a$ & $\mathrm{C}$ & B & $\mathrm{E}$ \\
\hline NWXJ28 & G. glabra & Xinjiang, Ziniquan & A & $3 b$ & A & A & E \\
\hline NWXJ32 & G. glabra & Xinjiang, Ziniquan & A & $3 b$ & A & A & $\mathrm{E}$ \\
\hline NWXJ25 & G. glabra & Xinjiang, Ziniquan & A & $3 b$ & A & A & E \\
\hline NWXJ30 & G. glabra & Xinjiang, Ziniquan & A & $3 b$ & A & A & E \\
\hline
\end{tabular}




$\begin{array}{cc}\text { A-B } & 3 c \\ \text { B } & 3 b \\ \text { F } & 5 \mathrm{a}\end{array}$

\begin{tabular}{ccc}
$\mathrm{A}$ & $\mathrm{B}$ & $\mathrm{A}-\mathrm{B}$ \\
$\mathrm{A}$ & $\mathrm{A}$ & $\mathrm{C}$ \\
$\mathrm{D}$ & $-* * *$ & $\mathrm{H}$ \\
\hline
\end{tabular}
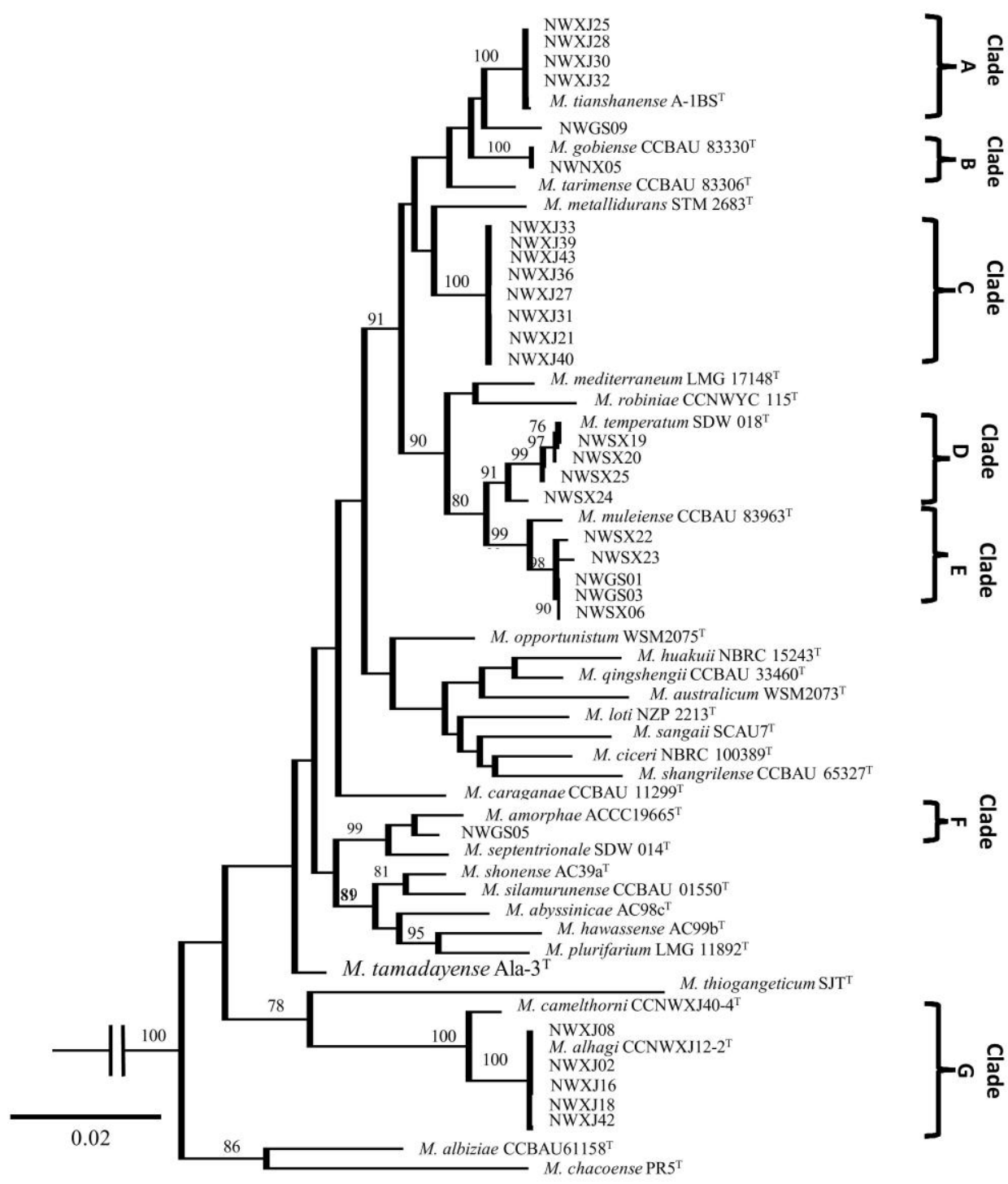

Figure 1. Phylogenetic tree of three concatenated housekeeping genes ( $r r s-r e c A-r p o B)$ of 59 mesorhizobial strains, constructed based on a maximum likelihood analysis. The genus name Mesorhizobium is abbreviated as $M$. The type strains are shown by a "T" at the end of each strain code. 